in the allocation of distinction awards can be also brought against the system.

AE carried out this study while a Harkness Fellow of the Commonwealth Fund of New York. We thank the Department of Health for providing us with the data on the NHS workforce.

Contributors: AE planned the study, carried out the analysis, and wrote the paper and acts as guarantor. HD collected the data from the various reports and prepared them for analysis. SE initiated the idea and participated in writing the paper. Funding: None

Conflict of interest: $\mathrm{AE}$ and $\mathrm{SE}$ are vice presidents of the Medical Practitioners Union, which is opposed to the present system of distinction awards for NHS consultants.

Advisory Committee on Distinction Awards. Report on the 1996 awards round. London: Department of Health, 1997.

2 Hospital Medical Staff: England and Wales. National and regional tables. Leeds: Statistics (Workforce) Branch, National Health Service Executive, 1996
3 Esmail A, Everington S. Racial discrimination against doctors from ethnic minorities. BMJ 1993;306:691-2.

Esmail A, Everington S. Asian doctors still being discriminated against. BMJ 1997;314:1619.

5 Esmail A, Everington S. Complaints may reflect racism. BMJ 1994;308:1374.

\section{Correction}

Comparison of blood or urine testing by patients with newly diagnosed non-insulin dependent diabetes: patient survey after randomised crossover trial

An error occurred in this paper by Pat Miles and colleagues (9 August 1997, pp 348-9). The first sentence of the final paragraph of the subjects, methods, and results section should read: "At 6 months, 42\% (47 [not 63]) opted for urine testing and 48\% (53 [not 72]) for blood testing; 10\% (11 [not 15]) chose both."

\title{
Association between use of a quilt and sudden infant death syndrome: case-control study
}

\author{
Anne-Louise Ponsonby, Terence Dwyer, David Couper, Jennifer Cochrane
}

The relation between an infant's sleeping environment and development of the sudden infant death syndrome depends on the infant's sleep position. ${ }^{1}$ We report how the association between the use of a quilt and the syndrome depends on sleep position.

\section{Subjects, methods, and results}

Between 1 October 1988 and 31 December 1995 in Tasmania 107 infants $<1$ year old died of the sudden infant death syndrome. Of the families affected, 100 $(93 \%)$ participated in this study and were compared directly with 196 age matched controls. Methods are described elsewhere. ${ }^{1}$ A quilt is a coverlet made by stitching two thicknesses of fabric together with a filling (usually synthetic) enclosed between the layers. Conditional multiple logistic regression ${ }^{2}$ was used to evaluate interaction effects; EGRET 0.26.6 software (Cytel Software, Cambridge, MA, USA) provided matched odds ratios with logit-based 95\% confidence intervals.

An adverse effect of quilt use was evident in infants who did not sleep prone but not in infants who slept prone (table). This interaction was not altered by adjustment for sleeping on sheepskin; the interaction between sleeping on sheepskin and sleeping prone; mattress liner; mattress type; use of a quilt under the infant; use of a pillow; infant illness; heating in the bedroom; amount of thermal insulation over the infant; maternal age; or family history of asthma. Adjustment for maternal smoking, breast feeding, or swaddling increased the size of the interaction.

A model was constructed to test whether the interaction was a marker for one of the previously identified modifiers of the prone position, ${ }^{1}$ with indicators for maternal smoking, use of a quilt, swaddling during last sleep, use of a natural fibre mattress, infant illness, and use of bedroom heating during last sleep; the interaction between the last five variables and the prone position was also evaluated. The interaction effect remained $(\mathrm{P}=0.01)$. An adverse effect of the use of a quilt was found among infants who did not sleep prone (adjusted odds ratio $6.16 ; 2.01$ to 18.87 ) but not among those who slept prone. Thus the interaction could not be explained by these factors. The interaction between sleep position and use of a quilt remained for infants and controls who slept in their own cot during the index sleep.

The adverse effect of quilt use among infants who did not sleep prone was greater in infants $\geqslant 12$ weeks old (odds ratio $6.77 ; 1.14$ to 40.21 ) than in younger infants (odds ratio 1.62; 0.27 to 9.59). Adjustment for being found prone reduced the effect of quilt use (adjusted odds ratio $3.16 ; 1.16$ to 8.60 ) suggesting that part of the adverse effect of quilts may occur by allowing infants to roll into the prone position. Among infants who did not sleep prone adjustment for swaddling, maternal smoking postnatally, breast feeding, and type of mattress gave an odds ratio of 5.63 (1.69 to 18.72) for quilt use. Review of the data at the infant's death scene showed that infants found supine with facial obstruction were reported by at least one witness to have had an overlying quilt. The risk of the sudden infant death syndrome for quilt use varied by usual sleep position (common odds ratio test

Relation between use of quilt and the sudden infant death syndrome according to usual sleeping position. Values are odds ratios ( $95 \%$ confidence intervals)

\begin{tabular}{lcc} 
Usual sleep position & $\begin{array}{c}\text { No quilt used during } \\
\text { last sleep }\end{array}$ & $\begin{array}{c}\text { Quilt used during } \\
\text { last sleep }\end{array}$ \\
\hline Supine or on side & 1.00 & $3.81(1.68$ to 8.65$)$ \\
\hline Prone & $9.19(3.24$ to 26.05$)$ & $9.53(3.52$ to 25.81$)$ \\
\hline
\end{tabular}

Menzies Centre for Population Health Research, University of Tasmania, GPO Box 252-23, Hobart, Australia 7001

Anne-Louise

Ponsonby, deputy director of research Terence Dwyer, director David Couper, biostatistician Jennifer Cochrane, research officer Correspondence to: Dr Ponsonby A.L.Ponsonby@ menzies.utas.edu.au

BMJ 1998;316:195-6 
$\mathrm{P}=0.017$ ) and was highest for infants found supine Unconditional analysis showed that quilt use attenuated the protective effect of sleeping supine compared with sleeping on the side (common odds ratio test, $\mathrm{P}=0.03$ )

\section{Comment}

Our data show that the use of a quilt increases the risk of the sudden infant death syndrome particularly among older infants who sleep supine or on their side; this is related to obstruction of the face by the quilt for some infants. At 16 weeks infants are able to pull clothing over their face. ${ }^{3}$ Infants $>3$ months old who sleep on their back or their side may be particularly likely to pull loose bedding over their faces; infants sleeping prone are less likely to be able to do this. The risk of the sudden infant death syndrome when a quilt is used (odds ratio 2.82; 1.95 to 4.08) is reduced after adjustment for factors that include the intermediate state of infants being found with their head under the covers. ${ }^{4}$ Duvets and quilts often do not tuck in because of their design. ${ }^{5}$ The recommendation that quilts should not be used in babies $<1$ year old is important for those who sleep supine or on their side. ${ }^{3}$

We thank the parents and infants who participated in this study the professional officers of the Tasmanian Ambulance Service for collecting data at the scene of death; the Departments of Hospital Pathology, Police, and Justice for their cooperation; Ego Pharmaceuticals for providing presents for control families after the home interview.

Contributors: A-L P initiated the study, designed the protocol, coordinated the collection of data, and undertook the statis- tical analysis, and participated in writing the paper. TD participated in the design of the study, was involved in the analysis and interpretation of data, and participated in writing the paper. DC participated in the statistical analysis and interpretation of data, and contributed to the writing of the paper. JC coordinated the fieldwork, data documentation, and quality control; participated in interpreting the data; and contributed to the writing of the paper. Allan Carmichael provided paediatric advice. John Williams provided computing advice. Helen Bain, Shirley Cameron, and Dianne McCracken were the principal research interviewers for collection of data. Janelle Booth prepared the manuscript for publication and participated in the literature search. A-L P is guarantor for the paper.

Funding: Tasmanian State Government, Australian Rotary Health Research Fund, National Health and Medical Research Council, National SIDS Council of Australia and its constituent organisations including the Sudden Infant Death Research Foundation, and Tasmanian Sanatoria After-Care Association. A-LP holds a National Health and Medical Research Council public health research and development fellowship.

Conflict of interest: None.

1 Ponsonby AL, Dwyer T, Gibbons LE, Cochrane JA, Wang YG. Factor potentiating the risk of sudden infant death syndrome associated with the prone position. N Engl J Med 1993;329:377-82.

2 Breslow NE, Day NE. Statistical methods in cancer research. Vol 1. Lyons: International Agency for Research on Cancer, 1980:196-279.

3 Illingsworth RS. The normal child: some problems of the early years and their treatment. 8th ed. London: Churchill Livingstone, 1983:16.

4 Fleming PJ, Blair PS, Bacon C, Bensley D, Smith I, Taylor E, et al. Environment of infants during sleep and risk of the sudden infant death syndrome: results of 1993-5 case-control study for confidential inquiry into stillbirths and deaths in infancy. BMJ 1996;313:191-5.

5 Wilson CA, Laing RM, Williams SM, Mitchell EA. Clothing and bedding and its relevance to sudden infant death syndrome: further results from the New Zealand cot death study. J Paediatr Child Health 1994;30:506-12.

(Accepted 11 June 1997)
See editorial by Metcalf

Department of Epidemiology Research, Danish

Epidemiology Science Centre,

Statens Serum

Institut, DK-2300

Copenhagen,

Denmark

Lise Lotte W

Nielsen,

medical student

Nete Munk Nielsen, physician

Mads Melbye,

professor

Peter Aaby,

professor

continued over

BMJ 1998;316:196-7
It has been suggested that people exposed to measles in utero may be at high risk of developing Crohn's disease in adulthood. ${ }^{12}$ Swedish investigators have reported an increased incidence of Crohn's disease in individuals born shortly after a measles epidemic, ${ }^{3}$ and they later described four pregnant women with measles, three of whose offspring developed Crohn's disease as adults. ${ }^{4}$

\section{Subjects, methods, and results}

To test this surprisingly strong association we conducted a record linkage study in Denmark. Hospital records from the Blegdamshospital (until 1976 the main hospital for treatment of infectious diseases in Copenhagen county) for 1915-66 were screened to identify pregnant women aged 15-43 years with measles. Their children were identified through the Copenhagen civil register (or church registers for those born before 1923) and linked through their civil registration system numbers with the national hospital discharge, cancer, and inflammatory bowel disease registries. Offspring who died before 31 December 1995 were identified through death certificates. For those with a gastrointestinal diagnosis, we contacted the patient's doctor for more information.

We identified 472 women aged 15 to 43 years who had been admitted with measles. Thirty three were pregnant: 11 developed measles during the first trimester, 9 during the second, 6 during the third, and 9 had exanthema less than 14 days after delivery. All but three women were identified in the civil registers. Four first trimester pregnancies were never registered and one child could not be found in any register.

Of the 26 offspring identified (including one set of twins), four died, one in infancy. The diagnoses of the other three, who died as adults, did not suggest inflammatory bowel disease (drug addiction, lung cancer, and heart disease). Among individuals still alive (median age 51.4 (36-79) years) none were registered as having Crohn's disease in either the inflammatory bowel 